\title{
RADIO CONTINUUM SURVEYS OF THE SKY
}

\author{
R. Wielebinski \\ Max-Planck-Institut für Radioastronomie \\ Auf dem Hügel 69 , D-5300 Bonn 1, F.R.G.
}

\section{INTRODUCTION}

Radio continuum surveys give us the fundamental information about the distribution of the radio intensity across the sky. The radio waves originate in three fundamental emitting processes and are measured superposed in the antenna beam. At the lowest radio frequencies (below 10 $\mathrm{GHz}$ ) the nonthermal emission process (synchrotron radiation) predominates. This radio emission comes from supernova remnants, from the "galactic background" (relativistic electrons diffusing in galactic magnetic fields), but also from extragalactic objects (galaxies, quasars, radio galaxies, etc.). In the frequency range $10 \mathrm{GHz}<\mathrm{f}<300 \mathrm{GHz}$ the thermal (free-free) process becomes dominant. In the Galaxy we see the HII regions along the galactic plane. In nearby galaxies we can identify the thermal emission in the disks. Above $300 \mathrm{GHz}$ the dust is seen. This is the boundary region between radio and far-infrared radiation.

\section{TECHNIQUES OF MAPPING THE SKY}

A radio continuum map is made using either a filled aperture radio telescope (e.g. paraboloidal dish, dipole array, etc.) or a synthesis radio telescope. In a filled aperture case the information about the intensity distribution is obtained by either scanning the sky with a fixed telescope or by driving the telescope in a T.V.-like raster fashion across the sky. In an aperture synthesis telescope the individual antennas track a position in the sky while the visibility functions ( $u-v$ data) for the different interferometers are recorded. Since in radio astronomy both amplitude and phase information is available a Fourier transfer of the $u-v$ data gives a radio map. With scanned telescope severe problems are encountered at high frequencies due to the effects of ground radiation and atmospheric noise. Various techniques have been developed to handle baseline problems. A filled aperture telescope allows, with care, the mapping of the whole sky. With the synthesis telescopes, which usually offer superior angular resolution, one of the major problems is the fact that a certain minimal distance of antenna spacings results in loss of information about large structures. 
The reduced radio continuum data comes in the form of arrays. These arrays are then easily transferable to modern image display systems. One of the most common mode of display is in the form of isodensity contours. For quick display a TV screen with a gray scale is often used. Colour displays, now commonly available, provide the spectacular maps of the sky.

\section{CONTINUUM SKY SURVEYS}

Since the pioneering work of Jansky and Reber numerous surveys have been published. As usual in astronomy the northern sky received more attention than the so important southern sky. The surveys at many frequencies steadily improved in angular resolution, sensitivity and in dynamic range. We distinguish between the all-sky surveys, the galactic plane surveys and the extended source surveys.

\subsection{All-sky Surveys}

The first attempt to 'join' a northern and southern survey was made by Dröge and Priester (1956). Combining two surveys the first all-sky map in galactic coordinates was produced. The problems of joining two surveys are not to be underestimated. A list of the 'current' all-sky surveys is given in Table 1 .

In this table the surveys with the best angular resolution at any one frequency are listed. These surveys are reasonably well 'matched' in spectral sensitivity, i.e. the sensitivity improves with frequency so that features with the spectral index $B=3.0\left(T \propto V^{-B}\right)$ are seen. The completion of the $2.7 \mathrm{GHz}$ survey to an all-sky northern survey is limited by sensitivity requirements - observing can be made only in good weather and without the deadly radar interference!

Table 1: All-sky Surveys

\begin{tabular}{|c|c|c|c|}
\hline Frequency & Beam & Coverage & Reference \\
\hline $30 \mathrm{MHz}$ & $11^{\bullet}$ & all sky & Crane, H.V. 1978, Australian J. Phys. $\underline{31}, 561$ \\
\hline $150 \mathrm{MHz}$ & $3: 5$ & all sky & $\begin{array}{l}\text { Landecker, T.L., Vielebinski, R. } 1970, \\
\text { Australian J. Phys. Suppl. } 16\end{array}$ \\
\hline $408 \mathrm{MHz}$ & $50^{\circ}$ & all sky & Haslam, C.G.T. et al. 1982, A\&A Suppl. 47,1 \\
\hline $1420 \mathrm{MHz}$ & $\begin{array}{l}35^{\prime} \\
35^{\circ} \\
30^{\prime}\end{array}$ & $\begin{array}{l}+90^{\circ}>\delta>+20^{\circ} \\
+20^{\circ}>\delta>-19^{\circ} \\
-15^{\circ}>\delta>-90^{\circ}\end{array}$ & $\begin{array}{l}\text { Reich, W. 1982, A\&A Suppl. } 48,219 \\
\text { Reich, P., Reich, W. 1986, A\&A Suppl. } 63,205 \\
\text { Loiseau, N., Reich, P. et al. (in prep.) }\end{array}$ \\
\hline $2.3 \mathrm{GHz}$ & $20^{\circ}$ & southern sky & Jonas, J.L. et al. 1985, A\&A Suppl. $\underline{62}, 105$ \\
\hline $2.7 \mathrm{GHz}$ & $20^{\circ}$ & $\begin{array}{l}\text { northern sky } \\
\text { (incomplete) }\end{array}$ & Reif, K., Reich, W., Steffen, P. (in prep.) \\
\hline $36 \mathrm{GHz}$ & $5^{\bullet}$ & all sky & $\begin{array}{l}\text { Strukov, I., Skulachev, D. 1986, Itogi Nauki } \\
\text { i Techniki, Astronomja, vol. 31， p.37 } \\
\text { Akademja Nauk CCCP }\end{array}$ \\
\hline
\end{tabular}




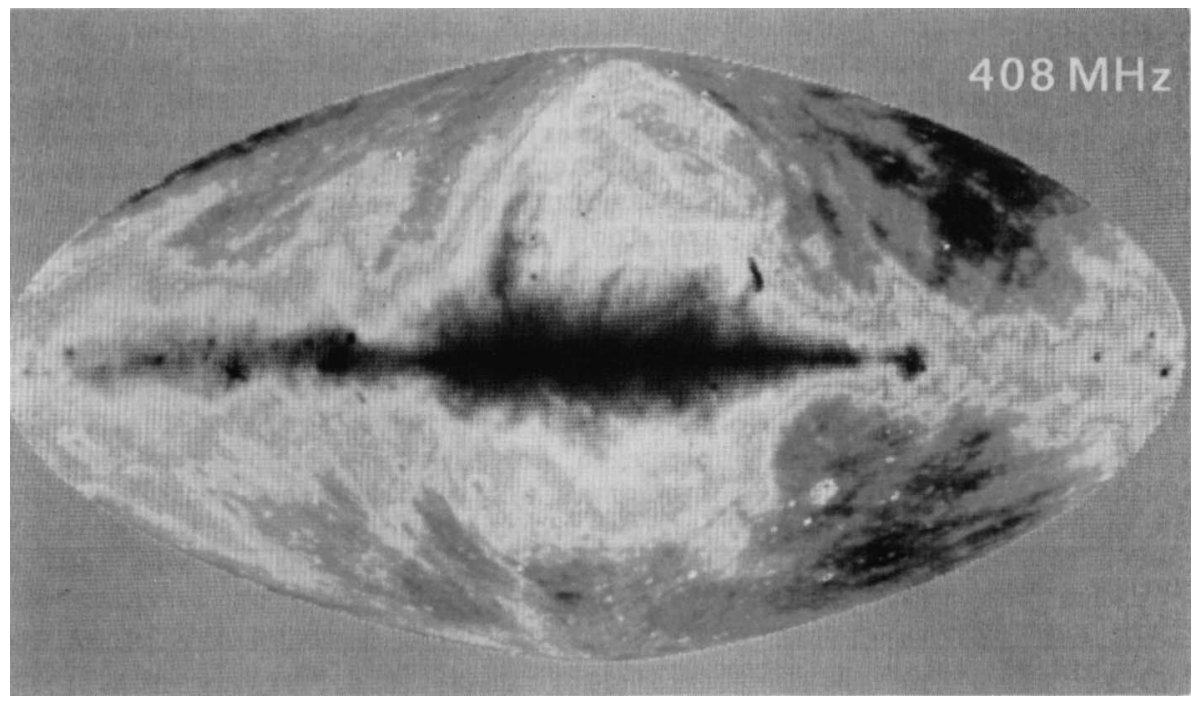

\subsection{Galactic Plane Surveys}

Most galactic sources like SNRs and HII regions are seen close to the galactic plane. Here good angular resolution plays a great role since many of the sources are only a few arc minutes in diameter. However not only the

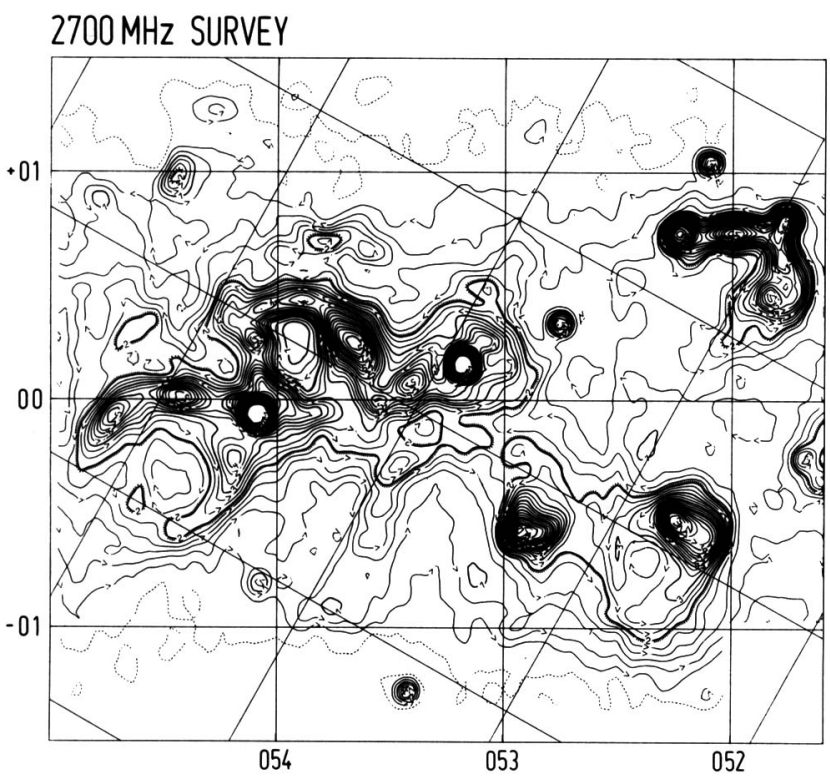


angular resolution but also the dynamic range are important. Since SNRs and HII regions are found often near each other it is necessary to 'see' the weak diffuse emission near an intense source. SNRs are also polarised. A list of selected galactic plane surveys is given in Table 2 . All the higher frequency surveys used full aperture radio telescopes. The extension of galactic plane surveys to higher frequencies or to better angular resolution goes only slowly. The amounts of observing time needed with the largest radio telescope are too large to give us significant new surveys in the near future. Only with new multibeaming techniques the speed of mapping may allow 1 arc min maps of a wide strip of the galactic plane. Here again the southern surveys lag behind the results of the northern sky. Polarisation surveys are in general not available.

Table 2: Selected Galactic Plane Surveys

\begin{tabular}{|c|c|c|c|c|c|c|}
\hline Frequency & Beam & & & Covera & & Reference \\
\hline 29.9 & 48 & $30^{\circ}$ & $\leftrightarrow$ & $225^{\circ}$ & $\pm 10^{\circ}\left(15^{\circ}\right)$ & $\begin{array}{l}\text { Jones, B.B., Finlay, E.A. 1974, } \\
\text { Australian J. Phys. 27, } 687\end{array}$ \\
\hline 408 & 3 & $55^{\circ}$ & $\leftrightarrow$ & $195^{\circ}$ & $\pm 3^{\circ}$ & $\begin{array}{l}\text { Green, A.J. 1974, A\&A Suppl. 18, } \\
267\end{array}$ \\
\hline 1405 & 9 & $162^{\circ}$ & $\leftrightarrow$ & $93^{\circ}$ & $\pm 4^{\circ}$ & $\begin{array}{l}\text { Kallas, E., Reich, W. 1980, A\&A } \\
\text { Suppl. } \underline{42}, 227\end{array}$ \\
\hline 1405 & 9 & $240^{\circ}$ & $\leftrightarrow$ & $162^{\circ}$ & $\pm 4^{\circ}$ & Reich, w. et al. (in prep.) \\
\hline 1410 & 14 & $356^{\circ}$ & $\leftrightarrow$ & $281^{\circ}$ & $+5^{\bullet}\left(6^{\bullet}\right)$ & $\begin{array}{l}\text { Hill, E.R. 1968, Australian J. } \\
\text { Phys. 21, } 735\end{array}$ \\
\hline 1414 & 10 & $55^{\circ}$ & $\leftrightarrow$ & $12^{\circ}$ & $\pm 4^{\circ}$ & $\begin{array}{l}\text { Altenhoff, W. et al. 1970, A\&A } \\
\text { Suppl. } 1,319\end{array}$ \\
\hline 2695 & 8 & $47^{\circ}$ & $\leftrightarrow$ & $286^{\circ}$ & $\pm 2^{\circ}$ & $\begin{array}{l}\text { Day et al. 1969-70, Australian } \\
\text { J. Phys. Suppl. 11, } 13\end{array}$ \\
\hline 2695 & 11 & $75^{\circ}$ & $\leftrightarrow$ & $345^{\circ}$ & $\pm 2^{\circ}$ & $\begin{array}{l}\text { Altenhoff, W. et al. 1970, A\&A } \\
\text { Suppl. } 1,319\end{array}$ \\
\hline 2695 & 4.4 & $76^{\circ}$ & $\leftrightarrow$ & $358^{\circ}$ & $\pm 1: 5$ & 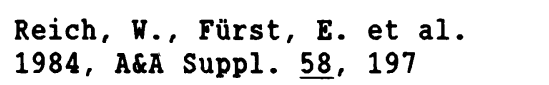 \\
\hline 2695 & 4.4 & $240^{\circ}$ & $\leftrightarrow$ & $358^{\circ}$ & $\pm 5^{\circ}$ & $\begin{array}{l}\text { Reich, W., Fürst, E. et al. (in } \\
\text { progress) }\end{array}$ \\
\hline 4875 & 2.6 & $60^{\circ}$ & $\leftrightarrow$ & $357: 5$ & $\pm 1^{\bullet}$ & $\begin{array}{l}\text { Altenhoff, w. et al. 1979, A\&A } \\
\text { Suppl. } 35,23\end{array}$ \\
\hline 5000 & 4.1 & $40^{\circ}$ & $\leftrightarrow$ & $190^{\circ}$ & $\pm 2^{\circ}$ & $\begin{array}{l}\text { Haynes, R.F. } 1978 \text {, Australian J. } \\
\text { Phys. Suppl. } \underline{45}\end{array}$ \\
\hline 10200 & 2.7 & $50^{\circ}$ & $\leftrightarrow$ & $355^{\circ}$ & $\pm 2^{\circ}$ & $\begin{array}{l}\text { Sofue, Y. et al. (partly avail- } \\
\text { able) }\end{array}$ \\
\hline
\end{tabular}




\subsection{Extended Source Surveys}

Both large filled aperture telescopes and synthesis instruments can be used to map selected well defined source types. Traditionally we had 'complete' surveys of HII regions (e.g. Felli and Churchwell, 1972). Complete surveys of SNRs do not exist. Every sensitive survey turns up new SNRs which are then usually followed up in individual papers. Also normal galaxies like M31, M33, etc. have been studied in individual papers. To follow up all the available data literature searches are necessary. The $100-\mathrm{m}$ telescope has been used to map most of the larger nearby galaxies in radio continuum. The angular resolution at $\lambda 2.8 \mathrm{~cm}$ is $\sim 1.2$. Comparable Westerbork data at $\lambda 49 \mathrm{~cm}$ and VLA ' $D$ ' array data at $\lambda 21 \mathrm{~cm}$ is also becoming available. Smaller galaxies have been studied and long flux lists are available in the literature. The 'Compendium' by Haynes et al. (1975) is a non cross-checked collection of various flux values. Radio galaxies have been first recognised in the Cambridge source surveys. A comprehensive study of the sources from the $3 \mathrm{C}$ list was made in Cambridge using the then new synthesis telescope (e.g. Mackay, 1969). Studies of southern radio galaxies go back even further - to the discovery and subsequent mapping of Centaurus A. The Molonglo cross radio telescope was used to produce a large catalogue of southern radio galaxies (Schilizzi and McAdam, 1975). With the present high resolution capabilities (down to arc sec) the VLA is the premier instrument to map radio galaxies. Numerous papers on various objects have been published. For the diffuse components of the radio galaxies single dish telescopes are preferable (e.g. Andernach and Wielebinski, 1982). Literature searches are necessary. A collection of older information on radio galaxies is given in Pacholczyk (1978). Clusters of galaxies are another source type being studied. Since

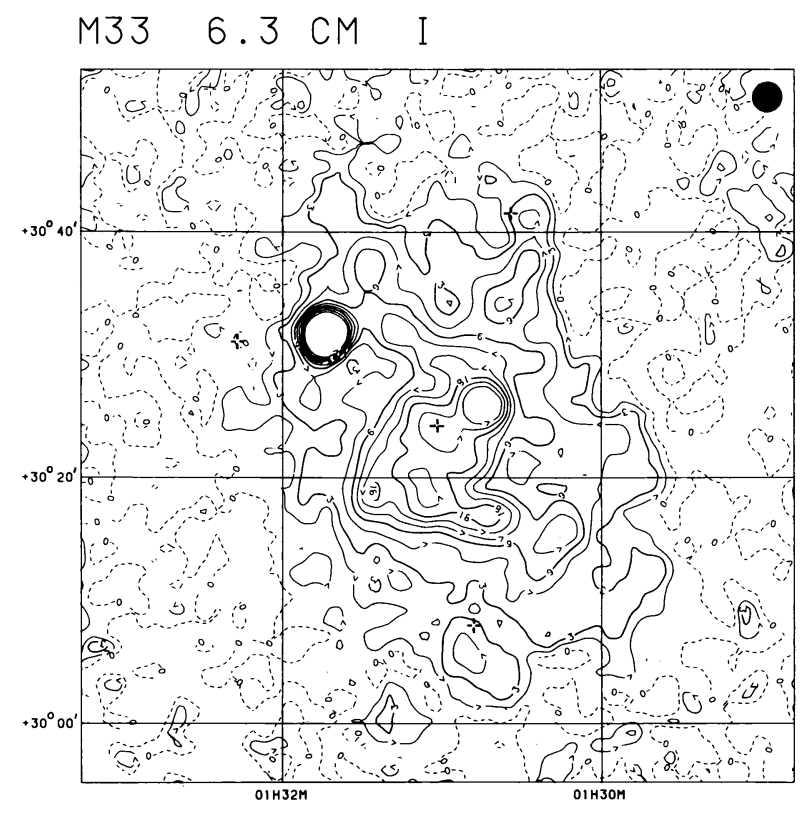


large fields have to be mapped large single dish telescopes but also synthesis instruments are used. Since steep spectrum sources are found in clusters low frequency investigations are often made. The Westerbork telescope produced a series of cluster papers based on $610 \mathrm{MHz}$ and $1.4 \mathrm{GHz}$ data. The Effelsberg work is contained in a series of papers giving results at 2.7 and $4.8 \mathrm{GHz}$ (e.g. Andernach et al., 1986). The literature on specific objects is growing rapidly all the time.

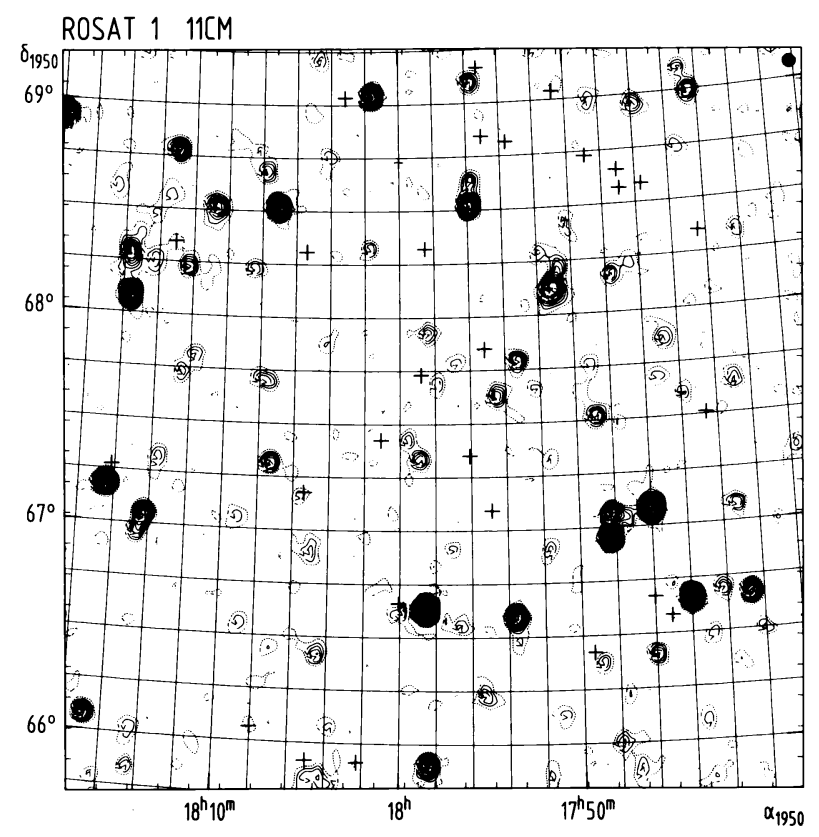

\section{POINT SOURCE SURVEYS}

The use of interferometers allowed the spectacular gathering of data about the distribution of 'point' sources across the sky. The early Cambridge surveys (2C, 3C, $4 \mathrm{C}$, etc.) and the southern counterparts (MSH surveys) gave us the basic information about discrete radio emitting regions. Many of the 'radio stars' of the early days turned out to be extended radio galaxies. Some of the radio sources remain unresolved even when studied with the VLBI methods.

It is less easy to give a table of references for point source surveys. The amount of gathered data is enormous and the completeness and reliability of these data vary enormously. An attempt by Dixon (1970) to produce a 'master list of radio sources' led to much confusion. No attempt was made to cross-check the data so that this master list remains a collection of a number of surveys.

A complete (all sky) cross-checked catalogue of sources with flux greater than $1 \mathrm{Jy}$ at $5 \mathrm{GHz}$ was published by Kühr et al. (1981a). This catalogue lists 518 sources which lie $\pm 10^{\circ}$ away from the galactic plane. 
Also the surrounding of the Magellanic Clouds is avoided. A further catalogue of sources by Kühr et al. (1981b) gives flux values for 1530 sources at several frequencies.

Other source surveys cover restricted areas but go down to a lower level. In the $5 \mathrm{C}$ series source detections are being made at $\sim 10 \mathrm{mJy}$ at $408 \mathrm{MHz}$. The Westerbork telescope was used to map several areas with ca. $1 \mathrm{mJy}$ noise. The RATAN 600 telescope in the Caucasus has made studies of several fields with source detections at $\sim 500 \mu \mathrm{Jy}$ at $4 \mathrm{GHz}$. The VLA has the best sensitivity and dramatic improvements in source surveys were made. In fact detection limits of $\sim 50 \mu \mathrm{Jy}$ and source count investigations down to $\sim 10 \mu \mathrm{Jy}$ have been published (Fomalont et al., 1984). As usual southern source studies lag behind but the use of the upgraded Molonglo Synthesis Telescope at $843 \mathrm{MHz}$ and of the now under construction Australia Telescope should change this picture.

\section{CONCLUSION}

The survey data which is available is very, very large. Some of the surveys are in machine-readable form. The distribution of these surveys for comparison has not so far been properly organised. It would be highly desirable to cross-check various surveys, to adjust calibrations and deposit this important data in a distribution centre. Only then would the astronomical community derive the benefit of the patient work of countless astronomers.

\section{REFERENCES}

Andernach, H., Wielebinski, R.: 1982, in "Extragalactic Radio Sources", IAU Symp. 95, eds.: D.S. Heeschen and C.M. Wade, Reidel Publ, Co., Dordrecht, p. 13

Andernach, H., Sievers, A., Kus, A., Schnaubelt, J.: 1986, Astron. Astrophys. Suppl. $\underline{65}, 561$

Dixon, R.S.: 1970, Astrophys. J. Suppl. 20, 1

Dröge, F., Priester, W.: 1956, Z. Astrophys. 40, 236

Felli, M., Churchwell, E.: 1972, Astron. Astrophys. Suppl. $\underline{5}, 369$

Fomalont, E.B., Kellermann, K.I., Wall, J.V., Weistrop, D.: 1984, Science $\underline{225}$, No. 4657,6 July, 23

Haynes, R.F., Huchtmeier, W.K.H., Siegman, B.C., Wright, A.E.: 1975, "A Compendium of Radio Measurements of Bright Galaxies, CSIRO, Melbourne

Kühr, H., Witzel, A., Pauliny-Toth, I.I.K., Nauber, U.: 1981a, Astron. Astrophys. Suppl. $\underline{45}, 367$

Kühr, H., Nauber, U., Pauliny-Toth, I.I.K., Witzel, A.: 1981b, MPIfR Preprint No. 55

Mackay, C.D.: 1969, Monthly Notices Roy. Astron. Soc. 145, 31

Pacholczyk, A.G.: 1978, "A Handbook of Radio Sources", Pachart, Tucson

Schilizzi, R.T., McAdam, W.B.: 1975, Memoirs Roy. Astron. Soc. $\underline{79}, 1$ 